\title{
Compliance with Intermittent Preventive Treatment Against Malaria in Pregnancy: Role of Health Center Quality and Accessibility in a Beninese Semi Urban Area
}

\author{
Padonou Sètondji Géraud Roméo ${ }^{1,}$, , Aguemon Badirou ${ }^{1}$, Damien Georgia ${ }^{2}$, Djossou Elisette ${ }^{1}$, \\ Codjia Estelle ${ }^{3}$, Hinson Antoine Vickey ${ }^{4}$, Ayelo Paul ${ }^{4}$ \\ ${ }^{1}$ Department of Public Health, Faculty of Health Sciences, University of Abomey-Calavi, Cotonou, Republic of Benin \\ ${ }^{2}$ Population and Health Department, Center for Training and Research in Population, University of Abomey-Calavi, Cotonou, Republic of \\ Benin \\ ${ }^{3}$ Teaching and Research Unit in Community Health and Epidemiology, Faculty of Health Sciences, University of Abomey-Calavi, Cotonou, \\ Republic of Benin \\ ${ }^{4}$ Research and Teaching Unit in Occupational Health and Environment, Faculty of Health Sciences, University of Abomey-Calavi, Cotonou, \\ Republic of Benin
}

\section{Email address: \\ geraudpad@yahoo.fr (P. S. G. Roméo) \\ ${ }^{*}$ Corresponding author}

\section{To cite this article:}

Padonou Sètondji Géraud Roméo, Aguemon Badirou, Damien Georgia, Djossou Elisette, Codjia Estelle, Hinson Antoine Vickey, Ayelo Paul. Compliance with Intermittent Preventive Treatment Against Malaria in Pregnancy: Role of Health Center Quality and Accessibility in a Beninese Semi Urban Area. European Journal of Preventive Medicine. Vol. 9, No. 1, 2021, pp. 8-13. doi: 10.11648/j.ejpm.20210901.12

Received: December 1, 2020; Accepted: December 9, 2020; Published: January 12, 2021

\begin{abstract}
Background: In a context of low coverage (around 40\%) of malarial intermittent preventive treatment in pregnant women in republic of Benin, we investigated the implication of health center quality and accessibility factors on the compliance with IPTp. Methods: In a cross-sectional study conducted from October 2017 to February 2018 in southern Benin, 422 women, pregnant in the last trimester or who gave birth less than a month ago were included. Conditions of access to the health center and factors related to the quality of services at the health center were collected. To search for associated factors, univariate analysis were performed using Chi2 (or Fisher's) and Student's test. Logistic regression model was fitted for multivariate analysis. Results: Rates of IPTp intake were $36.49 \%, 26.78 \%$ and $11.14 \%$ respectively for first, second and third doses. Two factors increased the probability of taking IPT: travel cost $(\mathrm{OR}=2.57[1.36 ; 3.92])$ and quality of reception at health center $(\mathrm{OR}=1.93[1.27 ; 2.64])$. Conversely, the increase in travel time from home to health center was associated with a lower probability of taking IPT $(\mathrm{OR}=0.91[0.85 ; 0.98])$. Conclusion: This study highlighted the need to take into account the improvement of the socioeconomic level of women and the improvement of the quality of services in health centers in order to achieve better coverage of IPT taking in pregnant women. Strengthening the health system in developing countries, in particular by recruiting health workers and training them, is beneficial.
\end{abstract}

Keywords: Pregnancy, IPT, Socioeconomic Level, Quality of Health Center Services

\section{Introduction}

Malaria is a serious pathology with more than 216 million cases recorded worldwide in 2016, of which sub-Saharan Africa is the most affected with nearly $90 \%$ of malaria-related deaths $[1,2]$. Every year, more than 25 million pregnant women are at risk of malaria worldwide and most live in sub-
Saharan Africa [1]. The populations most at risk are children under 5 years of age and especially pregnant women with negative consequences which are observed both in the mother and the fetus and have been widely described in the literature, in particular maternal anemia, and maternal death, fetal anemia, stillbirth, neonatal death, prematurity and intrauterine growth retardation leading to low birth weight. [2-11].

For antimalarial chemoprophylaxis in pregnant women, 
especially in endemic regions including sub-Saharan Africa, the World Health Organization (WHO) has recommended for more than a decade, the taking of intermittent preventive treatment (IPT) which is composed of a combination of sulfadoxine-pyrimethamine (SP) and now consists of taking supervised by a health worker from the 2 nd trimester at each antenatal consultation (ANC) according to the schedule established until childbirth while respecting an interval of at least one month between 2 consecutive intakes [12, 13].

The Benin Republic, a malaria endemic area, has adopted IPT in 2006 but national IPT coverage remains low and stands at $40 \%$. This rate is even lower in the Ouidah / Kpomassè / Tori-Bossito health zone, 30 to $35 \%$. Several parameters are involved in adherence to IPT and have been described in the literature. These include factors linked to women themselves, factors linked to the health system and the organization of care, factors linked to the availability and accessibility of care and drugs. In rural and semi-urban areas where health infrastructures are insufficient and where populations live in disadvantaged socioeconomic conditions, access to healthcare remains problematic.

The main objective of this study was to determine the role played by the quality of services at the health center and the conditions of accessibility in the adherence to taking IPT.

\section{Methods}

\subsection{Type, Area and Duration of Study}

We conducted a cross-sectional study from October 2017 to February 2018 in three towns Ouidah, Kpomassè and ToriBossito (OKT) located in a semi-urban area, south of the Republic of Benin. It's a tropical climate area where main mosquito vectors are Anopheles gambiae ss and Anopheles funestus. Plasmodium falciparum is the most commonly identified parasite species [14].

\subsection{Population}

Inclusion criteria:

1) Pregnant women in the last trimester

2) Women who gave birth less than a month ago

3) Permanent residence in the study area

Exclusion criteria:

1) Women in whom Sulfadoxine-Pyrimethamine was not recommended (allergy, taking cotrimoxazole in progress)

2) And those who refused to participate in the survey

To select the subjects, the first step was a stratified probability proportional sampling. We have formed 3 strata corresponding to Ouidah, Kpomassè and Tori-Bossito. The size within each stratum was proportional to the size of the population in the corresponding town. The second step was a 3 -stage cluster sampling. The first degree was the district of each town, the second degree the village and the third degree the house or the hearth. At each degree a random draw has been made. We used the Schwartz formula: $N=\varepsilon^{2} p q / i^{2}$ with $\varepsilon=1.96$ (for $\alpha=5 \%$ ), $p=$ Prevalence of the IPT in zone OKT $=35 \%, q=1-p=65 \%$, and $\mathrm{i}$ the expected accuracy $=0.05$.
We decided to increase the sample size by $20 \%$ to give greater statistical power. The calculated sample size was therefore 420 subjects. A total of 422 women were included in the study.

\subsection{Data Collection}

Each woman has been individually interviewed at the health center or at home. A questionnaire was filled in to collect data. The field team consisted of investigators trained and supervised by a senior epidemiologist.

\subsection{Variables Definition}

Dependent variable

Our dependent variable was taking IPT coded 1 when the woman took at least one dose of IPT during pregnancy and coded 0 if no.

Independent variables

These variables have been collected

1) Sociodemographic and economic factors: age, marital status, level of education, occupation status, ethnicity and religion.

2) Conditions of access to the health center: moving means, cost of travel, duration of travel.

3) Factors related to the quality of services at the health center: reception, waiting time and relationship with the health worker.

\subsection{Statistical Analysis}

After the field phase, all the survey forms have been verified to correct errors. We have adopted a 2-part strategy. The first part was the description of the socio-demographic factors of the study population. In Part 2, we investigated the factors associated with taking at least one IPT dose. For univariate analysis, Chi2 or Fisher test and Student test were used to compare percentages and means respectively. We performed multivariate analysis with a logistic regression model by including all variables with $p \leq 0.20$ in univariate step. Statistical significance was set at $\mathrm{p}<0.05$.

\subsection{Ethical Aspects}

Before the start of data collection, awareness and information sessions were organized for women. Free informed and oral consent was mandatory before inclusion in the study population. The anonymity of the data collected has been respected.

\section{Results}

\subsection{Description of the Study Population}

In our sample, $55.21 \%$ of women were at most 25 years old, mean age (SD) was 25.63 years (6.42). Most women were married $(80.09 \%)$, more than half were out of school $(57.35 \%)$. The artisans were in the majority $(45.97 \%)$, followed by merchants $(39.10 \%)$. There were several ethnic groups, the most represented of which was Fon (83.65\%). The dominant religion was Christian (60.90\%) (Table 1). 
Table 1. Description of the study population.

\begin{tabular}{ll}
\hline Variables & N (\%) or Mean (SD) \\
\hline Age & $25.63(6.42)$ \\
Age in class & \\
$\leq 25$ & $233(55.21)$ \\
] $25-35]$ & $154(36.49)$ \\
$>35$ & $35(8.29)$ \\
Marital status & \\
Married & $338(80.09)$ \\
Not married & $84(19.91)$ \\
Schooling level & \\
Unschooled & $242(57.35)$ \\
Primary and secondary & $175(41.47)$ \\
higher & $5(1.18)$ \\
Occupation & \\
Unemployed & $23(5.45)$ \\
Student & $27(6.40)$ \\
Artisan & $194(45.97)$ \\
Merchant & $165(39.10)$ \\
Civil servant & $13(3.08)$ \\
Ethnic group & \\
Fon & $353(83.65)$ \\
Toli & $29(6.87)$ \\
Mina & $40(9.48)$ \\
Religion & \\
Christian & $257(60.90)$ \\
Muslim & $39(9.24)$ \\
Traditional & $126(29.86)$ \\
\hline
\end{tabular}

\subsection{Doses of Malarial IPT During Pregnancy}

During their pregnancy, $25.59 \%$ of women never took IPT. Almost $75 \%$ took at least one dose of IPT. We have noticed a progressive decrease of taken doses of IPT during the pregnancy, $36.49 \%, 26.78 \%$ and $11.14 \%$ respectively for the 1 st dose, the 2nd dose and the 3rd dose. (Table 2)

\begin{tabular}{lll}
\hline Number of doses & $\mathbf{N}$ & $\mathbf{\%}$ \\
\hline 0 & 108 & 25.59 \\
1 & 154 & 36.49 \\
2 & 113 & 26.78 \\
3 & 47 & 11.14 \\
Total & 422 & 100 \\
\hline
\end{tabular}

\subsection{Conditions of Accessibility to Health Center}

Mean duration (SD) of travel from home to health center was 57.37 (1.87) minutes. And it was significantly shorter in women who complied with IPTp (54.93) than others (64.46), $(p=0.03)$. Less than half of the women, $44.55 \%$, went to the health center on foot and $54.74 \%$ spent less than 1000 FCFA as travel expenses. There was a significant association between travel cost and IPTp $(\mathrm{p}=0.001)$. Among women who took IPTp, those who spent less than 1000 FCFA were the most numerous. (Table 3 )

\subsection{Quality of Services at Health Center}

Majority of women, $98.10 \%$, reported being satisfied with the quality of the reception at the health center. This factor was significantly associated with the use of IPTp $(p=0.04)$. Among women who took IPTp, mostly were satisfied with the quality of the reception at the health center. Despite a mean waiting time of more than one hour (71.53 minutes), $86.49 \%$ of women did not consider the waiting time at the health center too long. The relationship between health workers and women was rated as good by the great majority of them. No significant associations were found between these factors and taking IPTp. (Table 3)

Table 2. Doses of malarial IPT during pregnancy.

Table 3. Conditions of accessibility and Quality of health center. Univariate analysis.

\begin{tabular}{|c|c|c|c|c|}
\hline & \multirow{2}{*}{ N (\%) or Mean (SD) } & \multicolumn{2}{|c|}{ Use of malarial IPTp (N or Mean) } & \multirow{2}{*}{$\boldsymbol{P}$} \\
\hline & & Yes & No & \\
\hline Travel duration (in minute) & $57.37(1.87)$ & 54.93 & 64.46 & 0.03 \\
\hline Going to health center on foot & & & & 0.25 \\
\hline Yes & $188(44.55)$ & 145 & 43 & \\
\hline No & $234(55.45)$ & 169 & 65 & \\
\hline Travel cost & & & & 0.001 \\
\hline$\geq 1000$ FCFA & $191(45.26)$ & 127 & 64 & \\
\hline$<1000$ FCFA & $231(54.74)$ & 187 & 44 & \\
\hline Quality of reception at health center & & & & $0.04^{\mathrm{a}}$ \\
\hline Satisfied & $414(98.10)$ & 307 & 107 & \\
\hline Not satisfied & $8(1.90)$ & 7 & 1 & \\
\hline Waiting time at health center (in minutes) & $71.53(3.17)$ & 71.19 & 73.52 & 0.85 \\
\hline Waiting time considered too long & & & & 0.13 \\
\hline Yes & $57(13.51)$ & 47 & 10 & \\
\hline No & $365(86.49)$ & 267 & 98 & \\
\hline Quality of relationship with health worker & & & & $0.57^{\mathrm{a}}$ \\
\hline Good & $411(97.39)$ & 305 & 106 & \\
\hline Bad & $11(2.61)$ & 9 & 2 & \\
\hline
\end{tabular}

${ }^{a}$ Fisher's test

\subsection{Factors Associated with the Use of IPTp. Multivariate Analysis}

The variables: travel's duration, travel's cost and the quality of reception at health center were significantly associated with taking IPT. The increase in the duration of the travel homehealth center reduced the probability of taking IPTp $(\mathrm{OR}=0.91$, $\mathrm{p}=0.003$ ). Conversely women who spent less than 1000 FCFA 
$(\mathrm{OR}=2.57, \mathrm{p}=0.02)$ and those who were satisfied with the quality of reception at health center $(\mathrm{OR}=1.93, \mathrm{p}=0.001)$ had a greater probability of taking IPTp compared to those who spent more than 1000 FCFA and those who were not satisfied with the quality of reception at health center. The time spent waiting at the health center did not seem to be a determining factor (Table 4).

Table 4. Factors associated with use of malarial IPTp. Logistic regression model.

\begin{tabular}{lll}
\hline \multirow{2}{*}{ Variables } & \multicolumn{2}{l}{ Use of malarial IPTp } \\
\cline { 2 - 3 } & Adjusted OR [95\% CI] & $\boldsymbol{p}$ \\
\hline $\begin{array}{l}\text { Travel duration (in minute) } \\
\text { Travel cost }\end{array}$ & $0.91[0.85 ; 0.98]$ & 0.003 \\
$\geq 1000$ FCFA & 1 & \\
$\quad<1000$ FCFA & $2.57[1.36 ; 3.92]$ & 0.02 \\
$\begin{array}{l}\text { Quality of reception at health center } \\
\text { Not Satisfied }\end{array}$ & 1 & \\
Satisfied & $1.93[1.27 ; 2.64]$ & 0.001 \\
Waiting time considered too long & & \\
No & 1 & \\
Yes & $0.86[0.31 ; 2.07]$ & 0.17 \\
\hline
\end{tabular}

\section{Discussion}

The main topic was to analyze factors related to pregnant women and their perceptions of the accessibility and the quality of services at the health center. In this study where 422 women were recruited in Ouidah-Kpomassè-Tori Bossito, objectives were to determine the compliance with the recommended doses of IPTp according to health center accessibility and quality.

Our results showed that the rates were $36.49 \%, 26.78 \%$ and $11.14 \%$ respectively for IPT1, IPT2 and IPT3. And $25.59 \%$ of women never took IPT during pregnancy. The probability of taking IPT was reduced when the travel duration from home to health center increased. Women who spent less than 1000 FCFA and those who declared to be satisfied with the quality of reception at health center were more likely to take malarial IPT during pregnancy.

Although some countries have better performance indicators, such as Ghana, where $98.5 \%$ of women received at least one dose of IPTp and 71\% received full IPTp of 3 doses [16], the coverage rate IPT in Benin is not far from what is observed in other African countries in general. The overall trend is towards low coverage. Indeed, according to the latest WHO Malaria Report 2017, the coverage rate of IPT3 was $19 \%$ on average in 23 African countries [1]. Both in West [15] and East Africa [17].

We assessed the quality of health center services through several variables including the quality of the reception, the quality of relationship between health worker and women, and the waiting time at the health center. Only the quality of reception at the health center was significantly associated with IPTp intake. In our study population, almost all women, more than $97 \%$, were satisfied with the reception at the health center and the relationships with the staff. Despite a mean waiting time of more than one hour, $86 \%$ of women did not consider this time too long. Several studies have demonstrated the benefit of good quality of services in health centers $[15,18$, 19], thus increasing attendance at these centers and women's adhesion. Health center managers and caregivers should therefore be encouraged to maintain good organization and quality of services in order to provide patients in general and pregnant women in particular with quality care [20].

In the univariate analysis of the conditions of access to the health center, we noted that cost of travel less than 1000 FCFA and reduced travel duration were positively related to taking IPTp. Same observations were made in the final multivariate model. Women who traveled shorter distances, therefore living closer to the health center, and women who spent less than 1,000 FCFA as travel cost, were more likely to have taken IPTp. These 2 variables are key parameters representing the accessibility of health centers. Displacement is an essential factor that can increase women's motivation to go to the health center if it is easy or discourages them to go if it is too restrictive. The duration taken to go to the health center, the time spent in the center and the time taken to return to the home are often perceived as penalizing compared to the rest of the professional activities generating income, or housewives to lead in the day. For women, going to the health center is perceived as time-consuming especially for antenatal care visits. In fact the doses of IPT are given during antenatal consultations according to a preestablished schedule. When they are not sick, consultation at health center is no longer a priority and becomes a secondary activity that they will do when they have time.

In addition, the financial factor plays an important role. There are 2 aspects to emphasize. First, populations living in rural or semi-urban areas such as OKT often have a low socioeconomic level, with limited resources. The cost of travel becomes important in the decision to go to the health center. Our study showed that women who visit the health center more often to receive IPTp spent less on travel costs than others. The low socioeconomic level is a limiting factor for taking IPTp as highlighted by a review of literature in 27 countries in sub-Saharan Africa [21] and more recently in Senegal [15] and Nigeria [22]. Secondly, women living in these areas are often poorly financially independent. For the most part, they depend on their husbands who provide them with the money they need to go to the health center. Our results therefore show the relevance of involving husbands in health issues concerning their wives. This finding has been made in other studies [23]. This strategy is beneficial in terms of public health, as shown in low-income countries [24-30].

Achieving the objectives of anti-malarial chemoprophylaxis in pregnant women is complex and requires action on several fronts. Especially in developing countries where several factors are involved. The good coordination of all these actions is a guarantee of success.

\section{Conclusion}

This study highlighted the need to take into account the improvement of the socioeconomic level of women. The promotion of income-generating activities among women 
living in disadvantaged areas in order to make them more financially independent, the development of socio-economic infrastructure are solution approaches Improvement of the quality of services in health centers and strengthening the health system in developing countries, in particular by recruiting health workers and training them, are beneficial in order to achieve better coverage of IPT taking in pregnant women.

\section{Declaration of Interest}

The authors declare that they have no competing interests.

\section{Acknowledgements}

We are grateful for all women who participate in this study. We thank the entire staff of field investigators.

\section{References}

[1] WHO. World Malaria Report 2017.

[2] WHO. World Malaria Report 2013.

[3] WHO. World Malaria Report. 2011.

[4] De Beaudrap, P., E. Turyakira, L. J. White, C. Nabasumba, B. Tumwebaze, A. Muehlenbachs, et al. Impact of malaria during pregnancy on pregnancy outcomes in a Ugandan prospective cohort with intensive malaria screening and prompt treatment. Malar J. 2013; 12: 139.

[5] Sohail, M., S. Shakeel, S. Kumari, A. Bharti, F. Zahid, S. Anwar, et al. Prevalence of Malaria Infection and Risk Factors Associated with Anaemia among Pregnant Women in Semiurban Community of Hazaribag, Jharkhand, India. Biomed Res Int. 2015; 2015: 740512.

[6] Takem, E. N. and U. D'Alessandro. Malaria in pregnancy. Mediterr J Hematol Infect Dis. 2013; 5 (1): e2013010.

[7] Hill, J., J. Hoyt, A. M. van Eijk, L. D'Mello-Guyett, F. O. Ter Kuile, R. Steketee, et al. Factors affecting the delivery, access, and use of interventions to prevent malaria in pregnancy in sub-Saharan Africa: a systematic review and meta-analysis. PLoS Med. 2013; 10 (7): e1001488.

[8] Bouyou-Akotet, M. K., D. P. Mawili Mboumba, E. Kendjo, F. Mbadinga, N. Obiang-Bekale, P. Mouidi, et al. Anaemia and severe malarial anaemia burden in febrile Gabonese children: a nine-year health facility based survey. J Infect Dev Ctries. 2013; 7 (12): 983-9.

[9] Kendjo, E., T. Agbenyega, K. Bojang, C. R. Newton, M. Bouyou-Akotet, F. Pedross, et al. Mortality patterns and site heterogeneity of severe malaria in African children. PLoS One. 2013; 8 (3): e58686.

[10] Desai, M., F. O. ter Kuile, F. Nosten, R. McGready, K. Asamoa, B. Brabin, et al. Epidemiology and burden of malaria in pregnancy. Lancet Infect Dis. 2007; 7 (2): 93-104.

[11] Eisele, T. P., D. A. Larsen, P. A. Anglewicz, J. Keating, J. Yukich, A. Bennett, et al. Malaria prevention in pregnancy, birthweight, and neonatal mortality: a meta-analysis of 32 national cross-sectional datasets in Africa. Lancet Infect Dis. 2012; 12 (12): 942-9.

[12] WHO. Updated WHO Policy Recommendation. 2012. Oct, Intermittent Preventive Treatment of malaria in pregnancy using Sulfadoxine Pyrimethamine (IPTp SP). 2012.

[13] WHO. Policy brief for the implementation of intermittent preventive treatment of malaria in pregnancy. World Health Organization. 2014; (1:12).

[14] Corbel, V., R. N'Guessan, C. Brengues, F. Chandre, L. Djogbenou, T. Martin, et al. Multiple insecticide resistance mechanisms in Anopheles gambiae and Culex quinquefasciatus from Benin, West Africa. Acta Trop. 2007; 101 (3): 207-16.

[15] Mbengue, M. A. S., A. K. Bei, A. Mboup, A. Ahouidi, M. Sarr, S. Mboup, et al. Factors influencing the use of malaria prevention strategies by women in Senegal: a cross-sectional study. Malar J. 2017; 16 (1): 470.

[16] Ibrahim, H., E. T. Maya, K. Issah, P. A. Apanga, E. G. Bachan, and C. L. Noora. Factors influencing uptake of intermittent preventive treatment of malaria in pregnancy using sulphadoxine pyrimethamine in Sunyani Municipality, Ghana. Pan Afr Med J. 2017; 28: 122.

[17] Ayubu, M. B. and W. B. Kidima. Monitoring Compliance and Acceptability of Intermittent Preventive Treatment of Malaria Using Sulfadoxine Pyrimethamine after Ten Years of Implementation in Tanzania. Malar Res Treat. 2017; 2017: 9761289.

[18] Orish, V. N., O. S. Onyeabor, J. N. Boampong, R. Afoakwah, E. Nwaefuna, S. Acquah, et al. Prevalence of intermittent preventive treatment with sulphadoxine-pyrimethamine (IPTp-SP) use during pregnancy and other associated factors in Sekondi-Takoradi, Ghana. Afr Health Sci. 2015; 15 (4): 1087-96.

[19] Nwaefuna, E. K., R. Afoakwah, V. N. Orish, A. Egyir-Yawson, and J. N. Boampong. Effectiveness of Intermittent Preventive Treatment in Pregnancy with Sulphadoxine-Pyrimethamine against Submicroscopic falciparum Malaria in Central Region, Ghana. J Parasitol Res. 2015; 2015: 959427.

[20] Namazzi, G., M. Okuga, M. Tetui, R. Muhumuza Kananura, A. Kakaire, S. Namutamba, et al. Working with community health workers to improve maternal and newborn health outcomes: implementation and scale-up lessons from eastern Uganda. Glob Health Action. 2017; 10 (sup4): 1345495.

[21] van Eijk, A. M., J. Hill, D. A. Larsen, J. Webster, R. W. Steketee, T. P. Eisele, et al. Coverage of intermittent preventive treatment and insecticide-treated nets for the control of malaria during pregnancy in sub-Saharan Africa: a synthesis and meta-analysis of national survey data, 2009-11. Lancet Infect Dis. 2013; 13 (12): 1029-42.

[22] Olukoya, O. O. and O. A. Adebiyi. Missed Opportunities for Intermittent Preventive Treatment for Malaria in Pregnancy in Nigeria: Evidence from Demographic and Health Survey in Nigeria 2013. Ann Ib Postgrad Med. 2017; 15 (2): 88-95.

[23] Kibusi, S. M., E. Kimunai, and C. S. Hines. Predictors for uptake of intermittent preventive treatment of malaria in pregnancy (IPTp) in Tanzania. BMC Public Health. 2015; 15: 540 . 
[24] Ampt, F., M. M. Mon, K. K. Than, M. M. Khin, P. A. Agius, C Morgan, et al. Correlates of male involvement in maternal and newborn health: a cross-sectional study of men in a peri-urban region of Myanmar. BMC Pregnancy Childbirth. 2015; 15: 122.

[25] Kaye, D. K., O. Kakaire, A. Nakimuli, M. O. Osinde, S. N. Mbalinda, and N. Kakande. Male involvement during pregnancy and childbirth: men's perceptions, practices and experiences during the care for women who developed childbirth complications in Mulago Hospital, Uganda. BMC Pregnancy Childbirth. 2014; 14: 54.

[26] Kululanga, L. I., J. Sundby, A. Malata, and E. Chirwa. Male involvement in maternity health care in Malawi. Afr J Reprod Health. 2012; 16 (1): 145-57.

[27] Kwambai, T. K., S. Dellicour, M. Desai, C. A. Ameh, B. Person, F. Achieng, et al. Perspectives of men on antenatal and delivery care service utilisation in rural western Kenya: a qualitative study. BMC Pregnancy Childbirth. 2013; 13: 134.

[28] Olayemi, O., F. A. Bello, C. O. Aimakhu, G. O. Obajimi, and A. O. Adekunle. Male participation in pregnancy and delivery in Nigeria: a survey of antenatal attendees. J Biosoc Sci. 2009; 41 (4): 493-503.

[29] Wai, K. M., A. Shibanuma, N. N. Oo, T. J. Fillman, Y. M. Saw, and M. Jimba. Are Husbands Involving in Their Spouses' Utilization of Maternal Care Services?: A Cross-Sectional Study in Yangon, Myanmar. PLoS One. 2015; 10 (12): e0144135.

[30] Pell, C., A. Menaca, F. Were, N. A. Afrah, S. Chatio, L. Manda-Taylor, et al. Factors affecting antenatal care attendance: results from qualitative studies in Ghana, Kenya and Malawi. PLoS One. 2013; 8 (1): e53747. 